ISSN: 2215-2644

revedu@gmail.com

Universidad de Costa Rica

Costa Rica

\title{
Masculinización de la matrícula universitaria en la carrera de Educación Física. Un análisis desde la perspectiva de género
}

Matus-Castillo, Carlos; Serra, Pedrona; Duclos-Bastías, Daniel; Castillo-Retamal, Franklin

Masculinización de la matrícula universitaria en la carrera de Educación Física. Un análisis desde la perspectiva de género

Revista Educación, vol. 46, núm. 1, 2022

Universidad de Costa Rica, Costa Rica

Disponible en: https://www.redalyc.org/articulo.oa?id=44068165025

DOI: https://doi.org/10.15517/revedu.v46i1.47576

\section{(c) $($ ) $\Theta \Theta$}

Esta obra está bajo una Licencia Creative Commons Atribución-NoComercial-SinDerivar 3.0 Internacional. 


\title{
Masculinización de la matrícula universitaria en la carrera de Educación Física. Un análisis desde la perspectiva de género
}

\author{
Male Dominant Enrollment among Physical Education Majors. An Analysis Based on Gender Perspective
}

Carlos Matus-Castillo

Universidad Católica de la Santísima Concepción, Chile

cmatus@ucsc.cl

(iD https://orcid.org/0000-0001-8019-6442

\section{Pedrona Serra}

Universitat de Barcelona y University of Balearic Islands,

España

pedronaserra@gencat.cat

(iD https://orcid.org/0000-0001-9527-9961

Daniel Duclos-Bastias

Pontificia Universidad Católica de Valparaíso, Chile

daniel.duclos@pucv.cl

(D) https://orcid.org/0000-0002-9020-5776

\section{Franklin Castillo-Retamal}

Universidad Católica del Maule, Chile

fcastillo@ucm.cl

(DD https://orcid.org/0000-0001-9118-4340

\author{
DOI: https://doi.org/10.15517/revedu.v46i1.47576 \\ Redalyc: https://www.redalyc.org/articulo.oa? \\ $\mathrm{id}=44068165025$
}

Recepción: 21 Julio 2021

Aprobación: 30 Agosto 2021

\section{Resumen:}

En Chile no se han desarrollado investigaciones sobre la formación del profesorado de Educación Física que consideren el análisis de la matrícula desde la perspectiva de género. Con el propósito de atender esta cuestión, el presente trabajo se ha fijado como objetivo describir, desde la perspectiva de género, el comportamiento de las matrículas femeninas y masculinas en los estudios de Pedagogía en Educación Física en las universidades chilenas, a partir de un enfoque cuantitativo, con diseño no experimental, retrospectivo y de alcance exploratorio-descriptivo, que analizó datos sobre la matrícula en Pedagogía en Educación Física en el periodo comprendido desde el año 2005 hasta 2019; se empleó información de fuentes oficiales de acceso abierto. Los resultados del análisis de las matrículas femeninas muestran un comportamiento que tiende a una disminución sistemática en la elección de las mujeres por esta carrera, lo que presenta una importante brecha negativa de género, que no es concordante con el comportamiento de las carreras del área pedagógica en general, ni con la matrícula de las instituciones de nivel superior. Así, se reporta una clara hegemonía de las matrículas masculinas para las carreras de Pedagogía en Educación Física en Chile.

Palabras Clave: Educación Física, Género, Estereotipos, Inscritos, Elección Profesional.

\section{ABSTRACT:}

Chile has not yet conducted studies on physical education teacher training programs which examine enrollment trends for that college major based on a gender approach. This study examines female and male enrollment trends for physical education teacher training programs throughought Chile's universities. The study was executed using a quantitative approach with a nonexperimental, retrospective and exploratory-descriptive design. Data pertaining to college training programs spanning 2005 to 2109 was used from official open-access sources. Study results of the study, reveal a reduction in the number of women that enroll to become PE Teacher-Training majors, thereby emphasizing a significant gender gap inconsistent with teacher training programs 
in other disciplines as well as student university enrollment, in general. This is a distinct reflection of the persistence of masculine hegemony in physical education teacher training programs in Chile.

Keywords: Physical Education, Gender, Stereotypes, Enrollment, Career Choice.

\section{INTRODUCCIÓN}

En Chile, la información que aporta el Sistema Nacional de Información de la Educación Superior (SIES) señala que la matrícula total femenina en educación superior (universitaria y técnico-profesional) registraba un total de 111.711 estudiantes en el año 1990, esto representaba el 44,8 \% del total; diez años después (2000), la matrícula femenina era de 213.347 , es decir, aumentó a un $47,2 \%$ su proporción ( $52,8 \%$ masculina). Las cifras expuestas reflejan información comprendida entre los años 1984 y 2009, donde el número de mujeres matriculadas en la educación superior chilena era menor a los hombres en los tres niveles formativos (pregrado, postgrado y postítulo) y en los tres tipos de instituciones del sistema de educación superior chileno: Institutos Profesionales (IP), Centros de Formación Técnica (CFT) y Universidades (SIES, 2010). Pese al bajo número de mujeres en la matrícula total en el periodo analizado por el SIES, se evidencia un aumento progresivo que tiene como hito el año 2009, donde la participación femenina supera, por primera vez, a la masculina (más del 50\%); hecho relevante en la educación superior chilena (Consejo Nacional de Educación de Chile [CNED], 2020). Recientemente, en los años 2018 y 2019 la matrícula total femenina llegó a un 52,9 \% y 53,0 \%, respectivamente (Ministerio de Educación de Chile [MINEDUC], 2019). Sin embargo, a pesar del aumento de las matrículas y de la finalización de las carreras por parte de las mujeres, la inserción laboral sigue mostrando falta de oportunidades (Organización para la Cooperación y el Desarrollo Económicos [OCDE], 2018; Ruiz-Gutiérrez y Santana-Vega, 2018).

Por áreas de conocimiento, el CNED (2016) señala que en el año 2016 las carreras que más presentaban brechas de género negativo en la matrícula total femenina eran las del área Tecnológica $(22,8 \%)$ y de las Ciencias (45,9\%). Por el contrario, donde existía más presencia femenina era en Salud (76,2 \%) y Educación $(72,6 \%)$.

En el caso puntual de las carreras del área Pedagógica (Matemática, Historia, Educación Física, entre otras), tuvieron como media un 66,6 \% y 66,9\% de matrícula femenina en los años 2018 y 2019, respectivamente; la Educación Parvularia (2018 y 2019: 99,8 \% media), Pedagogía en Educación Diferencial (2018: 95,9 \% y 2019: 95,6 \% medias) y Pedagogía en Educación Básica (2018: 84,7 \% y 2019: 84,1 \% medias) fueron las carreras con mayor proporción de matrícula femenina. Por el contrario, Pedagogía en Educación Física (PEF) presentaron la menor proporción de mujeres (2018: 29,9 \% y 2019: 32,3 \%) (CNED, 2020). Como se observa, este antecedente de PEF se contrapone a lo observado en las carreras pedagógicas, las cuáles, en su mayoría, a excepción de Pedagogía en Música, presentan una brecha positiva en la matrícula femenina. Otro antecedente relevante sobre la matrícula de PEF lo aportan Matus et al. (2021), quienes señalan que en el año 2018 esta carrera era una de las tres pedagogías que concentraban al 48,9\% de la matrícula total a nivel país en el área de educación.

De acuerdo con lo anterior y que PEF es una de las carreras chilenas que no evidencia estudios de matrícula con enfoque de género, sumado a que las instituciones educacionales forman de manera involuntaria o inconsciente en estereotipos sexistas (Garcia et al., 2012), y que el sistema educativo es un espacio de reproducción de las desigualdades de género (Marrero, 2019), es que se hace necesario contar con información sobre la situación histórica y actual del estudiantado y su evolución durante los últimos años. Por tanto, el objetivo del presente estudio es describir, desde la perspectiva de género, el comportamiento de las matrículas femeninas y masculinas en los estudios de Pedagogía en Educación Física en las universidades chilenas. 


\section{ESTADO DE LA CUESTIÓN Y PERSPECTIVAS TEÓRICAS}

Como se declara en el objetivo de la investigación, este artículo se posiciona desde la perspectiva de género para describir el comportamiento y situación de la matrícula de una carrera universitaria, por tanto, para efectos de este estudio, se comprenderá a la perspectiva de género como sinónimo de enfoque, visión o mirada de género o feminista, no exclusiva de las mujeres ni dirigida desde y hacia ellas. Esta perspectiva busca contribuir al fortalecimiento de un modelo democrático en el que la reivindicación de relaciones más justas beneficie tanto a hombres y mujeres, en toda su diversidad (Fontecha, 2006). Emplear el enfoque de género implica analizar críticamente las formas en que el sistema social delimita espacios y recursos para hombres y mujeres, condiciona sus elecciones; como el orden simbólico y real de género jerarquiza aquello considerado masculino por encima de aquello considerado femenino (Donoso y Velasco, 2013).

Bajo este marco, y en consideración del estereotipo de género como el papel (rol) que se configura con el conjunto de normas y prescripciones que dictan la sociedad y la cultura sobre el comportamiento femenino o masculino (Lamas, 2013), la literatura señala, por ejemplo, que los estereotipos de género podrían ser, desde la posición de las niñas, una amenaza ante la elección de cursar estudios en carreras masculinizadas, como las del área STEM (ciencias, tecnologías, ingenierías y matemáticas; altamente masculinizadas respecto a su matrícula), debido al temor que ellas piensan sobre sus habilidades y de lo que la sociedad piensa de ellas (Lane et al., 2011; Lorenzo et al., 2016). De acuerdo con lo expuesto, adquiere relevancia lo señalado por López et al. (2011), quienes indican que los estereotipos de género inciden mucho a la hora de escoger los estudios universitarios.

De forma puntual y de acuerdo con el objetivo de esta investigación, se ha indagado en la literatura sobre evidencias de la matrícula de PEF en otros contextos, puesto que en Chile no se reportan evidencias en esta línea. A raíz de lo anterior, se han identificado trabajos sobre las brechas de género en carreras de Ciencias de la Actividad Física y el Deporte (CAFD), Cultura Física y otras similares a PEF. En España, Serra et al. (2019a) publicaron datos sobre la presencia de mujeres en los estudios de CAFD en los dos campus del Instituto Nacional de Educación Física de Cataluña-INEFC (Lleida y Barcelona); reportan que en el curso 1999/2000 el $35 \%$ de la matrícula total era femenina, valor que muestra un descenso notable hasta el curso 2014/2015, donde se registró un 17,3\%. La investigación también revela que, entre las especialidades de Magisterio en Cataluña, la de Educación Física es la que posee una menor presencia femenina entre los cursos de los años 2000/2001 y 2011/2012, nunca superó el 50 \% de la matrícula total anual. A la vez, se informa que los centros privados muestran una mayor brecha de género y un mayor descenso en la matrícula femenina que los centros públicos. En línea con lo anterior, un trabajo realizado por Porto (2009), con datos desde el año 1993 a 2007 de tres universidades de Galicia (España), concluyó que, en consideración de todas las especialidades de Maestro/Magisterio, solo la especialidad de Educación Física (MEF) es la que no posee una razón de feminidad positiva en su matrícula, ya que los hombres casi duplican a las mujeres. También señala que la masculinización de las matrículas es más aguda en el caso de las licenciaturas sobre Actividad Física y Deporte. $\mathrm{Al}$ igual que los dos estudios anteriores, Garay et al. (2018) analizaron los datos de 30 años (1986/1987 a 2015/2016) de la matrícula de CAFD en la Universidad del País Vasco; advirtieron que existe un descenso en la media de la matrícula femenina durante los tres periodos analizados, ya que durante el primer periodo la Licenciatura en Educación Física (1986-2005) alcanzó un 32,4\%; posteriormente, la licenciatura en CAFD (2005-2010) descendió a un 24,4\%; y en el último periodo (2010-2016), las estudiantes matriculadas en el grado en CAFD llegaron solo a un 22,5\%. En Reino Unido, Elliott y Sander (2011) registran datos similares a los expuestos, dado que en el año 2009 se reportó solo un $32 \%$ de participación femenina en la matrícula de estudios relacionados al deporte. En esta misma línea, en Cuba también se han observado similitudes que se replican en las graduaciones de las carreras del área de Cultura Física, puesto que en el curso 2014/2015, del total de titulados, solo el 26,3\% eran mujeres (Quintana y Blazquez, 2017). 
Por otro lado, y a fin de comprender estos resultados, surgen investigaciones y teorías que colaboran en explicar cómo los estereotipos de género influyen en la toma de decisiones al momento de elegir estudios de nivel superior (López, 1993; Mosteiro-García, 1997; Ruiz-Gutiérrez y Santana-Vega, 2018), es el caso de la teoría del Aprendizaje Social (Bandura, 1987), la teoría de la Toma de Decisión Racional (Fonseca, 2013; Simon, 1997) y la teoría de la Acción Razonada (Fishbein y Ajzen, 1975; Reyes-Rodríguez, 2007), las cuáles serán analizadas en el apartado de la discusión.

En el marco de lo anterior, y desde un enfoque más bien político y de la relevancia que han adquirido los estudios de género, la Organización de las Naciones Unidas (ONU) reconoce que la población femenina (mujeres y niñas) corresponde a la mitad de la población mundial, por ende, la mitad del potencial humano para el desarrollo de la sociedad (ONU, 2015). Bajo esta lógica, la ONU aprobó la Agenda 2030 sobre el Desarrollo Sostenible, cuyo quinto objetivo propone lograr la igualdad entre los géneros y empoderar a todas las mujeres y niñas. Una de sus metas es aprobar y fortalecer políticas acertadas y leyes aplicables para promover la igualdad de género y el empoderamiento en todos los niveles de la población femenina (ONU, 2015). Al asociar lo expuesto con investigaciones sobre la educación con enfoque de género, y de acuerdo con el incremento de estos estudios en las últimas décadas, es que se ha logrado posicionar de forma paulatina el reconocimiento del género como construcción social, para afirmar su importancia en la sociedad, no así de las diferencias biológicas en el desarrollo más crítico y adecuado de las desigualdades entre los géneros en la Educación Física (Scraton, 1995). De esta forma, como señalan Alvariñas-Villaverde y Pazos-González (2018), la lucha contra los estereotipos de género en un campo tan concreto como la Educación Física requiere de un importante seguimiento de investigación, a fin de evidenciar el cumplimiento de metas, la verificación de la eficacia de los métodos y de las buenas prácticas. De esta forma, se hace necesario realizar constantemente revisiones y análisis sobre el cumplimiento de las metas que eliminen las diversas formas de discriminación hacia la mujer, como también evidenciar su participación en las distintas esferas de la sociedad con el propósito de lograr la igualdad y equidad de género. En el caso del sistema educativo, el cual posee un rol esencial en el proceso de socialización del género (Blanco et al., 2016), pues es un lugar de encuentro en diferentes etapas de las personas, lograr la igualdad puede traer beneficios que generen un impacto positivo en las generaciones actuales y venideras.

\section{MÉTODo}

\section{Tipo de estudio}

Este es un estudio con enfoque cuantitativo, puesto que se empleó el análisis de datos numéricos provenientes de fuentes oficiales. Cadena et al. (2017) señalan que una de las características de estas investigaciones es la medición penetrante, controlada y muy precisa, lo cual otorga objetividad y permite las generalizaciones. El diseño de la investigación es no experimental, ya que no existe manipulación de las variables, pues se presentan datos sin cambios intencionales (Hernández et al., 2014). Estos estudios también se clasifican según el momento en el cual los datos son recolectados en el tiempo, por tanto, esta investigación es de tipo longitudinal y retrospectiva, debido a que la información fue registrada desde el año 2005 al 2019. A la vez, es un estudio de alcance descriptivo y exploratorio (Hernández et al., 2014), ya que representa y analiza la matrícula femenina de PEF en las universidades chilenas en un marco carente de evidencias a nivel nacional.

\section{Universo}

Se comprende como universo o población al conjunto total de elementos que constituyen el ámbito de interés analítico y sobre el cual se establecerán las conclusiones de la investigación (López y Fachelli, 2015), por 
tanto, para este trabajo, el universo corresponde a todas las carreras de PEF chilenas y la información sobre su matrícula femenina y masculina (de primer año y total) desde el año 2005 hasta 2019. Cabe señalar que en el año 2019 se registraban un total de 31 carreras de PEF impartidas en 10 universidades estatales pertenecientes al Consejo de Rectores de Universidades Chilenas (CRUCH), seis a universidades privadas adscritas al CRUCH y 15 a universidades privadas no adscritas al CRUCH (CNED, 2020). Asimismo, es necesario precisar que las universidades estatales pertenecen al Estado de Chile y están adscritas al CRUCH; que las universidades privadas adscritas al CRUCH son instituciones reconocidas por el Estado bajo un rol público y no poseen fines de lucro y su financiamiento mayoritario es con recursos públicos; las universidades privadas no adscritas al CRUCH son instituciones educativas reconocidas por el Estado, pero su financiamiento es mayoritariamente privado (MINEDUC, 2020; Subsecretaría de Educación Superior de Chile, 2020).

\section{Fuente de información}

Se utilizó una base de datos oficial y abierta del centro de recursos en línea del CNED (2020), la cual cuenta con información procedente de las IES chilenas. Para acceder a la base de datos se empleó un navegador web y se ingresó durante el año 2020 a la dirección https://www.cned.cl/indices-educacion-superior; luego, se descargó el archivo Base indices Matrícula 2005-2019 en formato de hoja de cálculo Excel (.xls). Se realizó un análisis de datos secundarios con información de un reporte oficial; se seleccionaron solamente los datos de interés para este trabajo (Heinemann, 2008).

\section{Análisis de datos}

Se utilizó la estadística descriptiva; se estableció la distribución de las frecuencias de las matrículas mediante porcentajes y la media anual de matrícula de las carreras de PEF. La presentación de los resultados se realizó en una tabla y gráficos. Estos últimos incorporaron líneas de tendencia lineal, con el fin de representar, desde datos reales, posibles valores futuros de acuerdo con la tendencia.

Los datos fueron analizados según los siguientes periodos y criterios:

. 2005 a 2019: matrícula total y de primer año femenina y masculina de PEF de todas las universidades adscritas al CRUCH y de las universidades privadas.

- 2012 a 2019: matrícula de primer año femenina y masculina de PEF de todas las universidades en los cuatro años previo a la implementación de la gratuidad (2012-2015) y desde la implementación de la gratuidad (2016-2019). La implementación de la gratuidad el año 2016 (Ministerio de Hacienda de Chile, 2015) forma parte de los beneficios para estudiar carreras de educación superior; se asegura el financiamiento para el estudiantado perteneciente al $60 \%$ de la población más vulnerable del país. Cabe señalar que no todas las carreras PEF en Chile son susceptibles de la gratuidad, ya que, para acceder a ello, las universidades que imparten PEF deben cumplir con los requisitos contemplados en la Ley $n^{\circ} 21.091$ (MINEDUC, 2018), entre los que destacan: contar con acreditación institucional; ser instituciones sin fines de lucro; contar con un sistema de admisión transparente y sin discriminaciones, basado en el mérito, entre otros.

. 2005 a 2019: matrícula total femenina de PEF en carreras con modalidad de estudios diurnos y nocturnos. Las primeras se imparten durante el día, desde las 8:00 hasta las 19:00 horas, aproximadamente, y las de modalidad nocturna se dictan una vez terminadas las jornadas laborales. Los programas nocturnos se ofrecen como una oportunidad de acceso a la educación terciaria para personas que buscan compatibilizar trabajo y estudios (MINEDUC, 2015). 


\section{Resultados}

La Figura 1 presenta el periodo entre los años 2005 a 2019, en ella se observa una tendencia positiva (línea punteada) sobre el crecimiento de la matrícula femenina en el caso de las IES de forma agrupada (universidades, institutos profesionales y centros de formación técnica), con una media de 50,8\%, también en las universidades de forma particular ( $52 \%$ media) y, de manera sobresaliente, en las carreras del área Pedagógica $(66,9 \%$ media). Esto muestra un contraste cuando se les compara con PEF, puesto que los resultados revelan una tendencia decreciente en los 15 años estudiados, donde se tiene como valor más bajo un $28,5 \%$ en 2013. La media de PEF en este periodo fue de $31,2 \%$ de matrícula femenina.

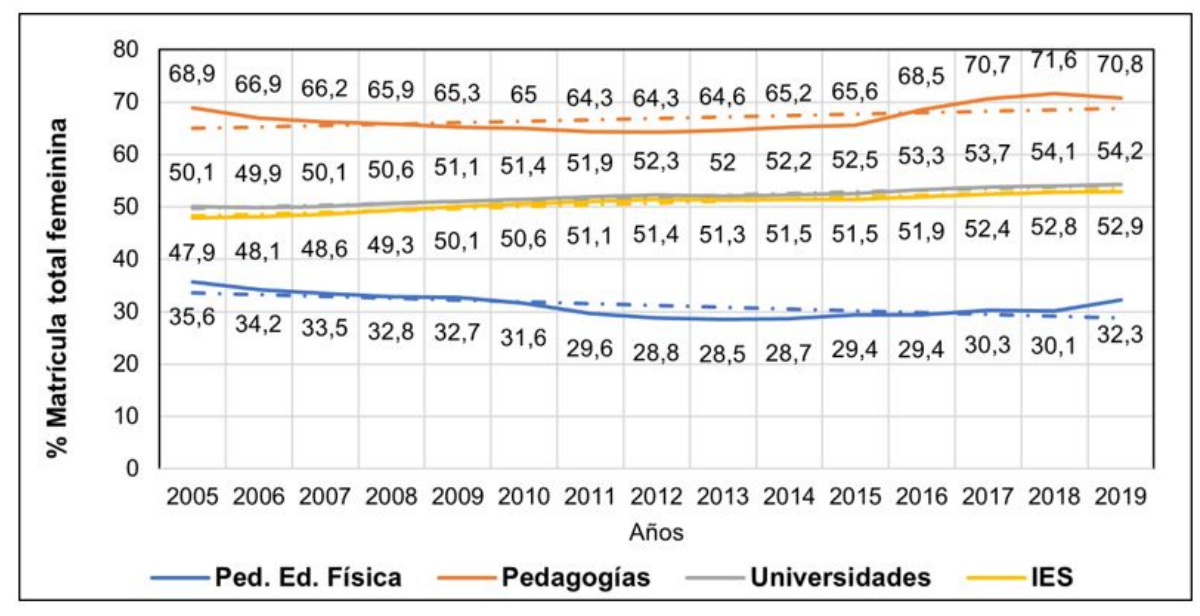

FIGURA 1

Porcentaje de matrícula total femenina en IES, universidades, pedagogías y PEF. Años 2005 a 2019. Fuente: elaboración propia con datos del CNED (2020).

La Figura 2 contiene los porcentajes de matrícula total femenina y masculina, considera todas las universidades que impartieron PEF entre los años 2005 a 2019. Los datos indican que la matrícula femenina ha estado por debajo de la masculina, lo que presenta una tendencia negativa. La matrícula femenina tuvo su valor máximo en el año 2005 (35,6\%). El gráfico muestra que entre los años 2011 a 2016 la matrícula femenina bajó del $30 \%$. Si se consideran los años extremos (2005 y 2019), se observa un descenso de 3,3\%, a la vez, la media para los 15 años fue de 31,2 \%, con una desviación estándar de 2,3 puntos y un rango de 7,2 puntos entre el valor máximo y el mínimo. 


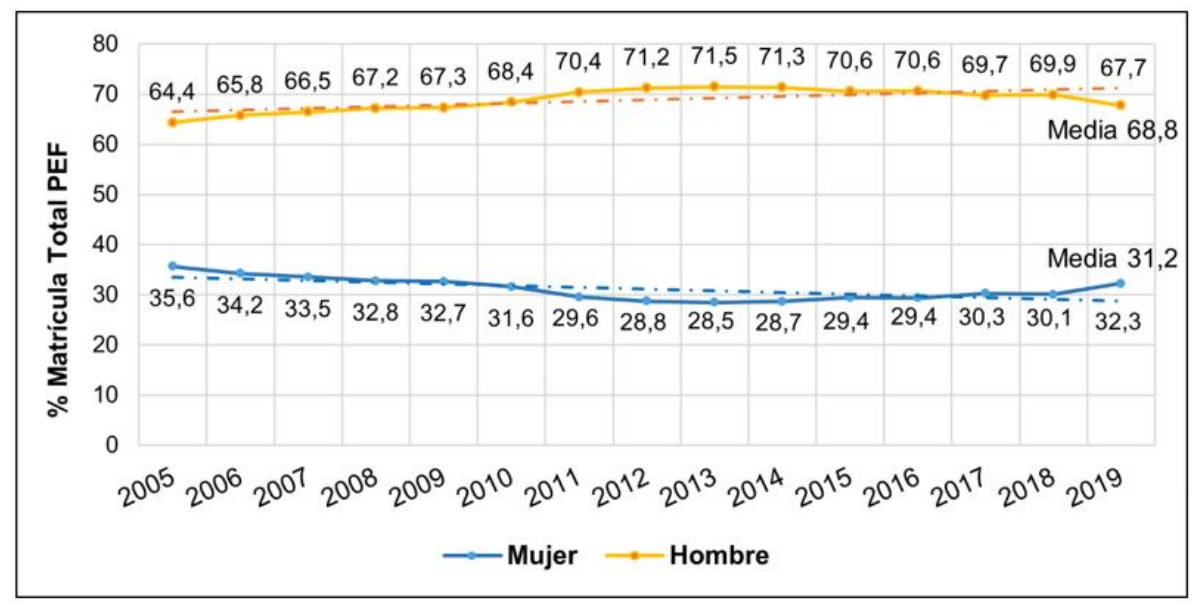

FIGURA 2

Porcentaje de matrícula total femenina y masculina de PEF. Años 2005 a 2019 Fuente: elaboración propia con datos del CNED (2020)

La Tabla 1 muestra el número total del estudiantado matriculado en PEF y la brecha de género (\%) en el periodo 2005-2019. La media de las estudiantes matriculadas no supera a la tercera parte del total del estudiantado durante los 15 años. El año 2013 se sitúa con la mayor brecha negativa (43,0 \%) y el año 2005 con la menor $(28,7 \%)$. Los resultados señalan que la brecha de género entre los años 2005 y 2019 registra una media de $37,7 \%$.

TABLA 1

Porcentaje de brecha de género y número de estudiantes en la matrícula total femenina y masculina de PEF. Años 2005 a 2019

\begin{tabular}{lllll}
\hline Año & Mujer (n) & Hombre (n) & Total (n) & Brecha \% \\
\hline $\mathbf{2 0 0 5}$ & 4.437 & 8.011 & 12.448 & 28,7 \\
$\mathbf{2 0 0 6}$ & 5.033 & 9.663 & 14.696 & 31,5 \\
$\mathbf{2 0 0 7}$ & 5.324 & 10.562 & 15.886 & 33 \\
$\mathbf{2 0 0 8}$ & 5.580 & 11.433 & 17.013 & 34,4 \\
$\mathbf{2 0 0 9}$ & 6.036 & 12.439 & 18.475 & 34,7 \\
$\mathbf{2 0 1 0}$ & 6.046 & 13.074 & 19.120 & 36,8 \\
$\mathbf{2 0 1 1}$ & 5.912 & 14.075 & 19.987 & 40,8 \\
$\mathbf{2 0 1 2}$ & 5.526 & 13.688 & 19.214 & 42,5 \\
$\mathbf{2 0 1 3}$ & 5.031 & 12.629 & 17.660 & 43 \\
$\mathbf{2 0 1 4}$ & 4.653 & 11.575 & 16.228 & 42,7 \\
$\mathbf{2 0 1 5}$ & 4.467 & 10.715 & 15.182 & 41,2 \\
$\mathbf{2 0 1 6}$ & 4.160 & 10.001 & 14.161 & 41,2 \\
$\mathbf{2 0 1 7}$ & 3.630 & 8.360 & 11.990 & 39,4 \\
$\mathbf{2 0 1 8}$ & 3.401 & 7.898 & 11.299 & 39,8 \\
$\mathbf{2 0 1 9}$ & 3.388 & 7.115 & 10.503 & 35,5 \\
\hline Media & $4.841,6$ & $10.749,2$ & $15.590,8$ & 37,7 \\
\hline
\end{tabular}

Fuente: elaboración propia con datos del CNED (2020).

La Figura 3 contiene la comparación de la matrícula total femenina de PEF entre las universidades pertenecientes al CRUCH y las universidades privadas que no pertenecen al CRUCH entre los años 2005 y 2019. Se observa que ambos tipos de universidades tienen una baja presencia de mujeres, esta es más significativa en las instituciones privadas (media de universidades CRUCH: 37,6\% v/s media universidades privadas: $28,7 \%$ ). Sin embargo, estas últimas muestran más estabilidad, ya que las pertenecientes al CRUCH presentan una baja continua y relevante entre los años 2009 y 2016. La desviación estándar fue de 4,2 puntos 
en las universidades del CRUCH y de 1,7 puntos en las privadas. El rango en las carreras del CRUCH es de 12,8 puntos y en las privadas de 5,1 puntos.

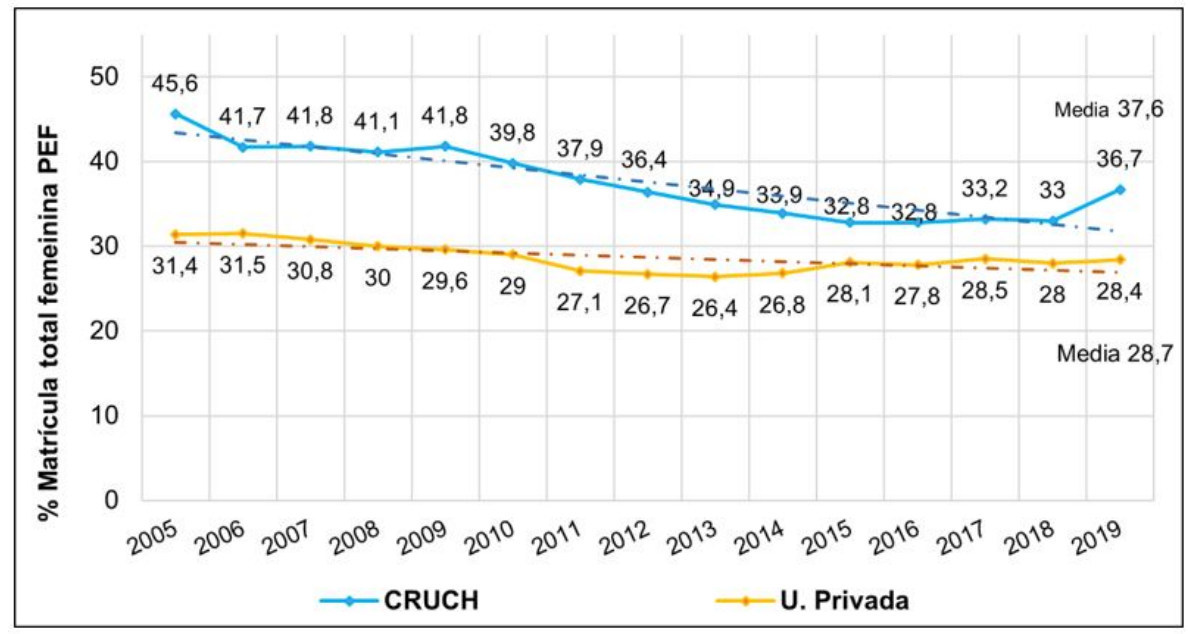

FIGURA 3

Porcentaje de matrícula total femenina de PEF en universidades del CRUCH y universidades privadas. Años 2005 a 2019

Fuente: elaboración propia con datos del CNED (2020).

A continuación, la Figura 4 muestra la matrícula femenina de PEF de $1^{\circ}$ año de todas las universidades en dos etapas. La primera es previa a la implementación del beneficio de la gratuidad (año 2012 a 2015) y la segunda es desde la gratuidad (año 2016 a 2019). De los ocho años analizados, en 2012 existe menor participación de mujeres, mientras que el 2017 (con gratuidad) registró el mayor porcentaje de matrícula de $1^{\circ}$ año, en donde se consideraron ambas etapas. Las cifras muestran una tendencia positiva durante los ocho años, con un aumento de 2,1 puntos porcentuales entre los años 2016 y 2017. Las medias de ambas etapas exponen que la participación femenina se ha incrementado en la etapa que considera la gratuidad (30,4 \% media).

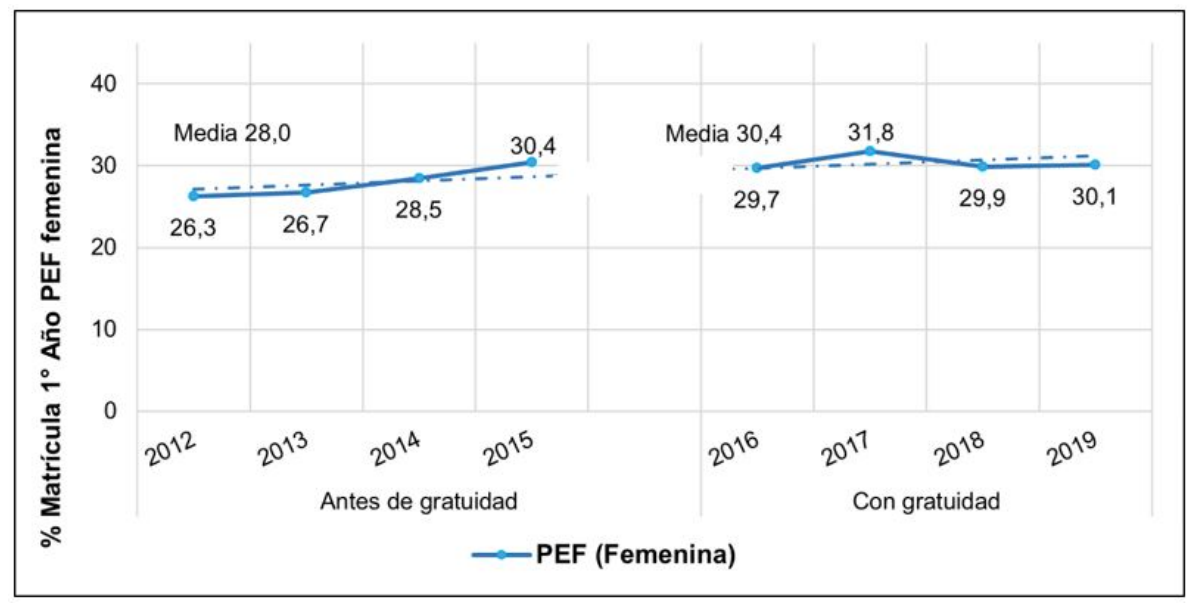

FIGURA 4

Porcentaje de matrícula femenina de $1^{\circ}$ año en PEF. 4 años previos y 4 años desde la implementación de la gratuidad. Años 2012 a 2019 Fuente: elaboración propia con datos del CNED (2020).

Los siguientes datos muestran la matrícula total femenina, comparan las jornadas diurnas y nocturnas en las cuales se dicta PEF en un periodo de 15 años (Figura 5). Las matrículas diurnas presentan una mayor 
proporción, con su máxima en 2005 (36,3 \%) y su mínima en 2013 (29\%) con una media de 31,6\%. Por el contrario, la jornada nocturna tiene menores proporciones de participación, pues obtiene su valor más alto en 2007 con 33,5 \%, y el valor mínimo en los años 2012 y 2014 con $22,5 \%$, su media es de $25,1 \%$. La tendencia para ambos casos es negativa y es más pronunciada en el régimen diurno.

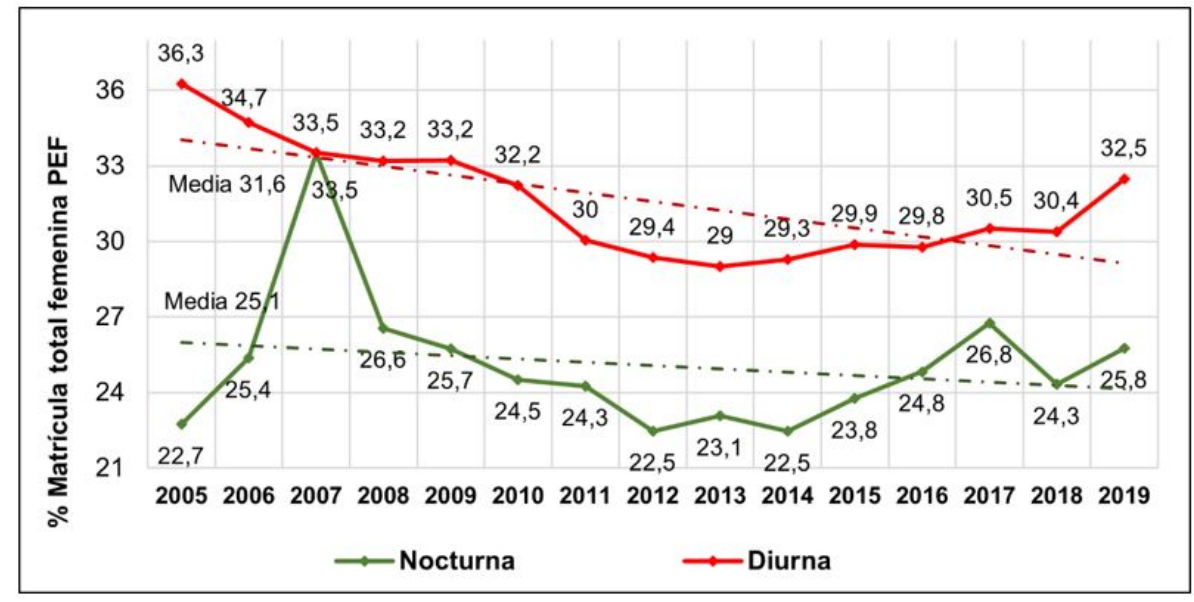

FIGURA 5

Porcentaje de matrícula total femenina en PEF para todas las universidades. Comparativa régimen diurno y nocturno. Años 2005 a 2019

Fuente: elaboración propia con datos del CNED (2020).

\section{Discusión}

Según los resultados de este trabajo, durante el año 2019 PEF se registró una matrícula total femenina de $32,3 \%$ y de $30,1 \%$ en el primer año, lo cual se contrapone con la situación de la mayoría de las carreras del área pedagógica en Chile (mayor matrícula femenina), que se extiende del año 2005 a 2019. Lo anterior muestra una conducta atípica en función de los valores de la matrícula femenina en las pedagogías, donde PEF tiene una mayor similitud al comportamiento de las matrículas de las carreras del área STEM, esto con base en los resultados de Mizala (2018), quien demostró que la matrícula de $1^{\circ}$ año de mujeres en carreras STEM no superó el $30 \%$ en el periodo 2007 - 2017 en Chile. En este sentido, una investigación realizada en España por Sáinz (2006) reportó que, dado múltiples factores asociados a los estereotipos de género y al proceso de socialización, las chicas tenían una menor intención de trabajar en algo relacionado con las nuevas tecnologías que los chicos; situación similar podría estar generándose en la Educación Física. Adicionalmente, la Organización de las Naciones Unidas para la Educación, la Ciencia y la Cultura (UNESCO, 2019) ha revelado que solo un $35 \%$ de estudiantes del área STEM son mujeres. Sumado a lo anterior, durante el periodo analizado en esta investigación (2005-2019), las matrículas femeninas totales en las IES chilenas muestran una tendencia positiva con una participación por sobre el $50 \%$ en media, lo cual es más prominente en las pedagogías. Sin embargo, esto contrasta con la situación de PEF, puesto que la brecha negativa de género se ha incrementado notoriamente desde el año 2005 a 2019.

Una forma de analizar los resultados se podría situar en los roles que tradicionalmente se han asignado a los hombres (productivos) y a las mujeres (reproductivos), en los que se acentúan las ideas de tareas masculinas y femeninas (Instituto Nacional de las Mujeres [INMUJERES], 2007). De esta manera, el género se transformaría en un factor determinante en el establecimiento de las relaciones sociales en la limitación de funciones y en la reproducción de patrones aceptados para ambos sexos (estereotipos de género), para influenciar de esta forma la toma de decisiones (Contreras et al., 2020; López, 1993; Ruiz-Gutiérrez y Santana-Vega, 2018). 
De forma más específica con PEF, un elemento que influiría en la elección o no de esta carrera, provendría desde las actitudes y roles desempeñados por los entrenadores y entrenadoras, y el profesorado de educación física, identificados en una investigación española como los principales agentes motivadores para la práctica de AF y deporte, a la vez, esto sirve como modelo de referencia para el estudiantado escolar (Serra, 2016). En este mismo sentido, se podría considerar la baja adherencia femenina a la práctica deportiva y de actividad física como un factor incidente respecto a la selección de cursar estudios de PEF (Porto, 2009). En el caso de Chile, los porcentajes de inactividad física femenina en población mayor y menor de edad es superior a la masculina (Ministerio del Deporte, 2018). Sin embargo, Garay et al. (2018) indican que la participación deportiva femenina, no sería una determinante a la hora de explicar por qué las mujeres no eligen los estudios de CAFD (en el caso de España) en una proporción similar que los hombres, puesto que, en las pruebas de acceso, las chicas muestran un mejor rendimiento físico que los varones y, por otro lado, las encuestas españolas de hábitos deportivos no muestran diferencias tan significativas entre hombres y mujeres. Lo expuesto invita claramente a reflexionar en torno al rol que desarrolla la educación física escolar (rol del profesorado, currículum oculto, contenidos, programas) y el sentido que adquiere la práctica deportiva y de actividad física previo a la elección de estudios de educación superior.

De forma concordante con lo señalado, en Chile existe evidencia de que las clases de educación física escolar se presentan con un enfoque androcéntrico, en donde se replican discursos y conductas sexistas que afectan a niñas y adolescentes (currículum oculto) (Poblete y Moreno, 2015; Rodríguez et al., 2017); existe separación de actividades por género, a pesar de que la educación física es mixta; también se reporta auto marginación por parte de las niñas y adolescentes, lo que otorga más protagonismo al género masculino en un ambiente donde el deporte y las prácticas competitivas generalmente se presentan como el principal contenido de las clases de educación física (Hidalgo y Almonacid, 2014). Sumado a lo anterior, en la formación del profesorado de Educación Física en las universidades, se mantiene un modelo deportivo masculino en los itinerarios formativos (Castillo et al., 2020), a pesar de que el Ministerio de Educación exige la incorporación de contenidos asociados al género y a la diversidad en carreras pedagógicas (Matus et al., 2021).

En este sentido, la teoría del aprendizaje social (Bandura, 1987), establece que la cultura y el comportamiento de una sociedad puede influir en el desarrollo y aprendizaje de una persona, así como también de forma recíproca de ella hacía otras (Bermeosolo, 2005). Esta teoría también permitiría entender el menor registro femenino en PEF, dado que las mujeres posiblemente no estarían ingresando a una disciplina que replica y privilegia estereotipos e intereses masculinos, por tanto, el comportamiento social podría limitarlas a participar de esta carrera. En concordancia con esto, la teoría de la toma de decisión racional (Fonseca, 2013; Simon, 1997) postula que las personas eligen los medios propicios para desarrollar y cumplir sus intereses, ello explicaría también la poca participación de mujeres en un medio (PEF) que no desarrolla o potencia sus intereses. De acuerdo con esta teoría, las elecciones se realizan de forma consciente e inconsciente en consideración de valores personales y el entorno social, lo que afectaría la elección de una carrera universitaria (Fonseca, 2013). Del mismo modo, la teoría de la acción razonada (Fishbein y Ajzen, 1975; Reyes-Rodríguez et al., 2007) relaciona el uso de información disponible sobre un tema para responder a una determinada conducta social. Es así como podría existir una influencia indirecta en la elección a participar como estudiante en PEF, dadas las informaciones, posiblemente masculinizadas, disponibles sobre esta carrera.

Como se ha señalado, en Chile no existen trabajos que analicen el comportamiento de la matrícula de PEF en perspectiva de género, por lo que se hace necesario observar y contrastar esta situación con otros contextos. Dentro de los hallazgos reportados por Serra et al. (2019a), se señala que la matrícula femenina en los estudios de actividad física y deporte y de Magisterio en Educación Física en Cataluña, España (1999-2014), siempre ha sido inferior a la masculina. Otro trabajo con resultados similares, realizado en España (1999 a 2007) (Porto, 2009), revela que las mujeres representan menos del tercio del estudiantado para CAFD, mientras que la media para MEF alcanzó un 36,4\%. Del mismo modo y también en España, Garay et al. (2018) dan 
a conocer la participación del estudiantado en CAFD (1986-2015) en tres etapas; la investigación reveló que la media de la matrícula femenina fue disminuyendo a través de los años: bajó en $10 \%$ desde la primera a la tercera etapa (cae de $32,4 \%$ a un $22,5 \%$ ). De esta forma, los hallazgos sobre la matrícula chilena son coherentes y responden a un comportamiento similar a las evidencias disponibles en la literatura consultada.

Por otro lado, los resultados de esta investigación determinaron que la matrícula femenina era menor en las universidades privadas no adscritas al Consejo de Rectores de Chile (CRUCH) que en las pertenecientes a este consejo. El comportamiento descrito es similar a lo reportado por Serra et al. (2019a) respecto a las matrículas en Cataluña, tanto en centros públicos y privados (1999 a 2014); se manifiesta que la brecha de género es mayor en estos últimos. La preferencia de las estudiantes chilenas por cursar PEF en instituciones públicas por sobre las privadas podría ser una respuesta a factores socioeconómicos, ya que las universidades públicas $(\mathrm{CRUCH})$ gozan de beneficios como la gratuidad y poseen más becas que las privadas, además muestran mayor prestigio.

Otro punto de análisis se relaciona con la implementación de la gratuidad (desde el año 2016). Los resultados de PEF mostraron un leve aumento de la matrícula femenina de primer año, que pasó de un $28 \%$ de media en los cuatro años previos a la implementación de gratuidad, a un 30,4\% en los cuatro años posteriores (2016 a 2019). Estas cifras son levemente superiores a las reportadas por carreras de Educación Física o similares impartidas en universidades públicas estatales mexicanas que poseen gratuidad y que pertenecen a la Asociación Nacional de Universidades e Instituciones de Educación Superior (ANUIES), donde se observa un promedio de participación femenina del 25,4 \% en el ciclo de ingreso 2019-2020 (ANUIES, 2021). A la vez, los datos chilenos se muestran más significativos al compararlos con la matrícula femenina ingresante a primer año 2020 de Educación Física en las universidades estatales y públicas de Costa Rica, donde la presencia femenina es solo del 17,9\% en comparación al 82,1 \% de matrícula masculina (Consejo Nacional de Rectores de Costa Rica, 2021).

De acuerdo con lo anterior, la gratuidad como beneficio estatal para el financiamiento de estudios superiores, se observa como una variable que podría estar ejerciendo una influencia positiva en el incremento de la matrícula; sin embargo, el poco tiempo de implementación de esta medida no permite realizar una proyección objetiva. Si bien el análisis de la gratuidad se enfocó en las matrículas de las carreras PEF y no pesquisó de forma personal a quienes poseen el beneficio de gratuidad, existe información que reporta que esta política pública ha afectado positiva y significativamente en el incremento de la participación femenina en los estudios de nivel superior en Chile (ONU Mujeres, 2020).

El análisis comparativo entre las jornadas en las cuales se dicta PEF, mostró que las matrículas femeninas diurnas presentan una mayor proporción que en la modalidad nocturna. Sobre la tendencia, para ambos casos esta es negativa, más pronunciada la baja de matrícula en el régimen diurno y más estable en la modalidad nocturna. Este análisis lleva a precisar que, en el estudiantado chileno, mujeres y hombres que participan de carreras en jornadas nocturnas tiene características diferentes a quienes asisten en jornada diurna, ya que se observa una mayor proporción de hombres que de mujeres (caso contrario se observa en la modalidad diurna) y, en general, tienen 25 o más años; no ingresan directamente desde la secundaria y, desde su egreso escolar, han transcurrido en promedio tres o más años (Arancibia y Trigueros, 2018). Otro aspecto es que mayoritariamente trabajan para financiar sus estudios y requieren de más apoyo pedagógico y académico (Segobia, 2014). Cabe señalar, que las universidades que dictan PEF en modalidad nocturna son principalmente privadas no adscritas al CRUCH (CNED, 2020), por tanto, tienen menos beneficios estatales. De acuerdo con lo expuesto, se puede señalar que los resultados obtenidos son coherentes con el comportamiento de las matrículas femeninas de carreras universitarias chilenas reportadas en el Informe GET: Género, Educación y Trabajo (Comunidad Mujer, 2016), ya que también existen proporcionalmente menos mujeres estudiando en modalidad nocturna que aquellas que lo hacen en el día. No obstante, se debe considerar que las brechas de género de PEF en jornada nocturna son más agudas en todas sus comparaciones con las matrículas de las IES (universidades, IP y CFT). Uno de los motivos que se reportan en este informe 
sobre la menor participación femenina se asocia a las costumbres y concepciones arraigadas que consideran que las mujeres no pueden o no deben salir por las noches, junto a razones de seguridad personal (Comunidad Mujer, 2016). A ello se suman las múltiples barreras sociales y culturales, a las cuales se ven enfrentadas las mujeres y que ya se han mencionado anteriormente.

En suma, la influencia de la familia y el entorno cercano; las expectativas de la sociedad sobre los roles de mujeres y hombres; los estereotipos de género; el enfoque androcéntrico y la preponderancia de contenidos deportivos y poco cercanos a las niñas, junto con la hegemonía masculina en las clases de educación física; la inserción y oportunidades laborales; la proyección de la satisfacción de objetivos profesionales y personales, podrían tener una preponderancia en la selección de una carrera universitaria, en este caso de PEF. Con ello se demuestro una problemática de tipo estructural y cultural desde la perspectiva de género (Elliott y Sander, 2011; Garay et al., 2018; Horne, 1999; Kirk y Oliver, 2014; López, 1993; Matus et al., 2021; Porto, 2009; Serra et al., 2019b; Serra et al., 2019a).

De acuerdo con los aportes de Porto (2009) y Serra et al. (2016), los elementos señalados previamente respecto a la influencia en la elección de PEF, podrían agruparse en las siguientes categorías: el desarrollo de procesos de dualización de género que afectan a la Educación Física; el desarrollo de una Educación Física androcéntrica focalizada en el deporte y alejada de gustos de niñas y adolescentes; la existencia de una cultura femenina que no se considera en las clases de Educación Física ni en la formación del profesorado de la especialidad; las representaciones colectivas y prejuiciadas acerca de los roles de mujeres y hombres en la sociedad y el desinterés femenino por las prácticas físicas y deportivas en general. De esta forma, se releva la necesidad de incorporar la perspectiva de género de forma transversal en las universidades, dado que son espacios públicos que, mediante sus dinámicas, tienen una alta incidencia e impacto en la sociedad (AcuñaRodríguez, 2014).

\section{Conclusiones}

El análisis de las matrículas de PEF muestra un comportamiento que tiende a una disminución sistemática en la elección femenina por esta carrera; presenta una importante brecha negativa de género que no es concordante con la conducta de las carreras del área pedagógica, ni con la matrícula femenina de las instituciones de educación superior en Chile. En este sentido, PEF más bien responde a lo observado en carreras del área STEM. En vista de la clara hegemonía de las matrículas masculinas para PEF en Chile. Según la literatura sobre el comportamiento del estudiantado universitario, PEF puede ser considerada como una carrera masculinizada desde la perspectiva de su composición de matrícula. Serían múltiples los factores de influencia en la elección o no de las mujeres por estudiar PEF, los cuales se asociarían principalmente a la creencia de que es una carrera masculinizada, lo cual se originaría en la educación física escolar, en el círculo familiar y de amigos, en la misma educación superior (universidad) y en el ámbito laboral (educativo y deportivo).

Cabe señalar que es preocupante y desconocido el impacto socioeducativo que se estaría generando en la Educación Física escolar y en el sistema deportivo a raíz de los estereotipos, el sexismo y el machismo, producto de la baja presencia de profesoras de la especialidad.

Respecto a las recomendaciones que aporta la literatura para promover el incremento de la matrícula en esta carrera, surgen con mayor frecuencia: la revisión y ajustes a los programas de estudio de PEF con perspectiva de género, los cuales deberían potenciar contenidos asociados a la salud, a aspectos sociales y que releven la Educación Física escolar; incluir imágenes femeninas (de deportistas, por ejemplo) en los materiales pedagógicos; desarrollar materiales de sensibilización para adolescentes, familias y equipos de orientación educativa; generar instancias formativas en cuestiones de género para el profesorado universitario y de educación escolar; promover la generación de evidencia científica que realce la perspectiva de género en las investigaciones asociadas a la Educación Física; luchar contra el currículum oculto en la formación del 
profesorado con estrategias que permitan promover una educación equitativa en clave de género (AlvariñasVillaverde y Pazos-González, 2018; Areces et al., 2011; Delfy, 1998; Hartmann-Tews, 2011; Petry y Brackenridge, 2001; Porto, 2009; Serra et al., 2016).

Los resultados del estudio permiten establecer líneas de investigación futuras a fin de visualizar, comprender y relevar las problemáticas de género que se presentan en PEF. De esta forma, se sugiere analizar la existencia o no de brechas de género considerando otras variables complementarias a la matrícula, como es el caso de las tasas de titulación oportuna, las tasas de aprobación y reprobación de las asignaturas, las tasas de retención y deserción de los estudios. También se recomienda realizar investigaciones bajo un enfoque cualitativo, ya que permitirían conocer los perfiles del estudiantado, identificar y explicar los elementos facilitadores y obstaculizadores, y las motivaciones de las mujeres por la elección o no de cursar estos estudios. Este mismo enfoque podría aplicarse al profesorado universitario para analizar, desde un punto de vista didáctico o metodológico, la existencia de ciertos comportamientos o técnicas que podrían influir en la masculinización de los estudios y su matrícula. Por otro lado, también se sugiere investigar los programas de estudios y planificaciones de las clases de PEF, a fin de examinar si existen componentes asociados a la perspectiva de género.

Los hallazgos de esta investigación pueden ser empleados por las universidades y el Ministerio de Educación, a fin de diseñar políticas y estrategias que permitan incrementar la participación femenina en los estudios de PEF y, a la vez, reducir los múltiples impactos producto de las brechas de género detectadas.

\section{Agradecimientos}

Este artículo se ha elaborado durante el desarrollo del proyecto: La perspectiva de género en la Pedagogía en Educación Física. Estado, enfoques y propuestas, para y desde la Formación Inicial Docente, financiado por ANID / CONICYT / FONDECYT / INICIACIÓN / N Proyecto 11190524, Chile.

\section{ReFERENCIAS}

Acuña-Rodríguez, M. (2014). Reflexiones en torno al vínculo género-educación superior. Revista Educación, 38(2), 89-106. https://doi.org/10.15517/revedu.v38i2.15263

Alvariñas-Villaverde, M. y Pazos-González, M. (2018). Estereotipos de género en Educación Física, una revisión centrada en el alumnado. Revista electrónica de investigación educativa, 20(4), 154-163. https://doi.org/10.243 20/redie.2018.20.4.1840

Arancibia, R. y Trigueros, C. (2018). Aproximaciones a la deserción universitaria en Chile. Educação e Pesquisa, 44. h ttps://doi.org/10.1590/s1678-4634201708165743

Areces, A., Mosquera, M. y Martin, R. (2011). 25 Anos da Faculta de Ciencias do deporte e a Educación Física/INEF de Galicia (1986-2011). Universidade da Coruña.

Asociación Nacional de Universidades e Instituciones de Educación Superior [ANUIES]. (2021). Anuarios Estadisticos de Educación Superior. http://www.anuies.mx/informacion-y-servicios/informacion-estadistica-deeducacion-superior/anuario-estadistico-de-educacion-superior

Bandura, A. (1987). Teoria del aprendizaje social. Espasa-Calpe.

Bermeosolo, J. (2005). Cómo aprenden los seres humanos: Una aproximación psicopedagógica. Ediciones UC. https://ww w.digitaliapublishing.com/a/38222/como-aprenden-los-seres-humanos---una-aproximacion-psicopedagogica

Blanco, M., Sánchez, P. y Ramos, F. (2016). Conciliación de la Vida Laboral y Familiar en Mujeres en Formación Ocupacional. Multidisciplinary Journal of Educational Research, 6(2), 127-151. https://doi.org/10.17583/rem ie.2016.1795 
Cadena, P., Rendón, R., Aguilar, J., Salinas, E., de la Cruz, F. y Sangerman, D. (2017). Métodos cuantitativos, métodos cualitativos o su combinación en la investigación: Un acercamiento en las ciencias sociales. Revista Mexicana de Ciencias Agricolas, 8(7), 1603-1617. https://doi.org/10.29312/remexca.v8i7.515

Castillo, F., Almonacid, A., Castillo, M. y Oliveira, A. (2020). Formación de profesores de Educación Física en Chile: Una mirada histórica. Retos, (38), 317-324. https://doi.org/10.47197/retos.v38i38.73304

Comunidad Mujer. (2016). Género, Educación y Trabajo. La brecha persistente. Primer estudio sobre la desigualdad de género en el ciclo de vida. Una revisión de los últimos 25 años. Comunidad Mujer Chile. http://www.informeget .cl/wp-content/uploads/2016/06/Informe-GET.pdf

Consejo Nacional de Educación de Chile [CNED]. (2016). Tendencias Índices 2016. Consejo Nacional de Educación. https://www.cned.cl/sites/default/files/tendencias_indices_junio_2016.pdf

Consejo Nacional de Educación de Chile [CNED]. (2020). Índices Base de Datos Matrícula 2005-2019. Consejo Nacional de Educación. https://www.cned.cl/bases-de-datos

Consejo Nacional de Rectores de Costa Rica. (2021). Perfil sociodemográfico de la matrícula deprimer ingreso del primer periodo lectivo, del Sistema de Educación Superior Universitaria Estatal, 2014 - 2020. https://siesue.conare.ac.c $\mathrm{r} /$ matricula-de-primer-ingreso-matricula-de-i-periodo-lectivo/

Contreras, S., Sarmiento, C., Cárcamo, L. y Huerta, N. (2020). Diagnóstico de las condiciones de base para la incorporación de perspectiva de género en la carrera de Periodismo de la Universidad Austral de Chile (Valdivia). Comunicación y Género, 3(1), 17-26. https://doi.org/10.5209/cgen.67499

Delfy, L.A. (1998). Career opportunity in sport. Journal of Physical Education, Recreation \& Dance, 69(7), 17-22. ht tps://doi.org/10.1080/07303084.1998.10605588

Donoso, T. y Velasco, A. (2013). ¿Por qué una propuesta de formación en perspectiva de género en el ámbito universitario? Profesorado. Revista de curriculum y formación del profesorado, 17(1), 71-88. https://revistaseug.u gr.es/index.php/profesorado/article/view/19448

Elliott, D. y Sander, L. (2011). Why females don't do sport degrees. Journal of Hospitality, Leisure, Sports and Tourism Education, 10(1), 85-98. https://www.proquest.com/openview/578a4eb76c58aa2e27dd9be4373bfd00e/1?pqorigsite $=$ gscholar\&cbl $=40023$

Fontecha, M. (2006). Intervención didáctica desde la perspectiva de género en la formación inicial de un grupo de docentes en educación Física. [Tesis doctoral]. Universidad del País Vasco. https://repositorio.minedu.gob.pe/handle/20 $.500 .12799 / 1831$ ?show $=$ full

Fishbein, M. y Ajzen, I. (1975). Belief, Attitude, Intention and Behavior: An Introduction to Theory and Research. Reading, Mass.

Fonseca, C. (2013). Toma de Decisión: ¿Teoría racional o de racionalidad limitada? Kálathos. Revista Transdisciplinaria Metro-Inter, 7(1).http://kalathos.metro.inter.edu/kalathos_mag/publications/archivo5_vo 17_no1.pdf

Garay, B., Elcoroaristizabal, E., Vizcarra, M., Prat, M., Serra, P. y Soler, S. (2018). ¿Existe sesgo de género en los estudios de ciencias de la actividad física y el deporte? Retos, (34), 150-154. https://doi.org/10.47197/retos.v0i34.58846

Garcia, C., Larena, R. y Miró, I. (2012). Participación de las «Otras Mujeres» en las Escuelas: Superando Estereotipos de Género y Mejorando el Aprendizaje. Multidisciplinary Journal of Educational Research, 2(1), 37-55. https:/ /doi.org/10.4471/remie.2012.02

Hartmann-Tews, I. (2011). Gender issues at the German Sport University. Jornada Dones i esport: bones pràctiques en recerca, docència i innivació, Grup d'Investigació Social i Educativa de l'Activitat Física i l'Esport, INEFC Barcelona. https://cutt.ly/eWqzQa6

Heinemann, K. (2008). Introducción a la metodología de la investigación empirica en las ciencias del deporte. Editorial Paidotribo.

Hernández, R., Fernández, C. y Baptista, M. (2014). Metodología de la investigación (6ta ed.). McGraw-Hill / Interamericana Editores.

Hidalgo, T. y Almonacid, Al. (2014). Estereotipos de género en las clases de educación física. Journal of Movement and Health (JMH), 15(2), 85-96. https://dialnet.unirioja.es/servlet/articulo?codigo=6348118 
Horne, J. (1999). Understanding Sport: An Introduction to the Sociological and Cultural Analysis of Sport. Routledge, London.

Instituto Nacional de las Mujeres [INMUJERES]. (2007). ABC de género en la Administración Pública (2da ed.). INMUJERES - PNUD. http://cedoc.inmujeres.gob.mx/documentos_download/100903.pdf

Kirk, D. y Oliver, K. L. (2014). La misma historia de siempre: reproducción y reciclaje del discurso dominante en la investigación sobre la educación física de las chicas. Apunts. Educación Física y Deportes, 116, 7-22. https://doi. org/10.5672/apunts.2014-0983.es.(2014/2).116.01

Lamas, M. (2013). El género: La construcción cultural de la diferencia sexual (4ta ed.). Miguel Ángel Porrúa. http://re positorio.ciem.ucr.ac.cr/jspui/handle/123456789/154

Lane, K., Goh, J. y Driver-Linn, E. (2011). Implicit science stereotypes mediate the relationship between gender and academic participation. Sex Roles, 66, 220 - 234. https://doi.org/10.1007/s11199-011-0036-z

Lorenzo, M., Álvarez-Lires, F., Álvarez-Lires, M., Serrallé-Marzoa, J. (2016). La amenaza del estereotipo: elección de estudios de ingeniería y educación tecnocientífica. Opción, 32(9), 54-76. https://produccioncientificaluz.org/in dex.php/opcion/article/view/21715

López, M. (1993). La elección de una carrera típicamente femenina o masculina desde la perspectiva psicosocial: La influencia del género [Tesis doctoral]. Universidad Nacional de Educación a Distancia. https://redined.mecd.g ob.es/xmlui/handle/11162/84699

López, P. y Fachelli, S. (2015). Metodología de la investigación social cuantitativa. Universidad Autónoma de Barcelona. http://tecnicasavanzadas.sociales.uba.ar/wp-content/uploads/sites/156/2020/08/A04.02-Roldany-Fachelli.-Cap-3.6-Analisis-de-Tablas-de-Contingencia-1.pdf

López, M., Puertas, S. y Sáinz, M. (2011). Why don't girls choose Technological Studies? Adolescents' stereotypes and attitudes towards studies related to Medicine or Engineering. The Spanish Journal of Psychology, 14(1), 74-87. $\mathrm{h}$ ttps://doi.org/10.5209/rev_sjop.2011.v14.n1.6

Marrero, G. (2019). La perspectiva de género: una reivindicación necesaria en el ámbito educativo. Revista Educación, 43(2), 643-658. https://doi.org/10.15517/revedu.v43i2.32426

Matus, C., Cornejo, M. y Castillo, F. (2021). La perspectiva de género en la formación inicial docente en la Educación Física chilena. Retos, 40, 326-335. https://doi.org/10.47197/retos.v1i40.83082

Ministerio del Deporte. (2018). Resumen Ejecutivo: Encuesta Nacional de Hábitos de Actividad Física y Deporte en Población de 18 años y más. https://cutt.ly/0WqQtIw

Ministerio de Educación de Chile [MINEDUC]. (2015). Evolución de oferta y matrícula 2007-2014: Educación superior vespertina en Chile. https://bit.ly/3wIq2ex

Ministerio de Educación de Chile [MINEDUC]. (2018). Ley 21091. Sobre Educación Superior. Congreso Nacional. https://www.bcn.cl/leychile/navegar?idNorma $=1118991$

Ministerio de Educación de Chile [MINEDUC]. (2019). Informe matricula 2019 en Educación Superior en Chile. Ministerio de Educación de Chile. http://bibliotecadigital.mineduc.cl//handle/20.500.12365/4608

Ministerio de Educación de Chile [MINEDUC]. (2020). Consejo de Rectores. https://www.ayudamineduc.cl/ficha/ consejo-de-rectores-5

Ministerio de Hacienda de Chile. (2015). Modifica La Ley n 20.882 de presupuestos del sector público del año 2016. https://bit.ly/2VKxtoL

Mizala, A. (2018). Género, cultura y desempeño en matemáticas. Anales de la Universidad de Chile, (14), 125-150. h ttps://doi.org/10.5354/0717-8883.2018.51143

Mosteiro-García, M. (1997). El género como factor condicionante de la elección de carrera: Hacia una orientación para la igualdad de oportunidades entre los sexos. Revista Galego-Portuguesa de Psicoloxia e Educación, 1, 305-315. ht tps://ruc.udc.es/dspace/handle/2183/6622

Organización para la Cooperación y el Desarrollo Económicos [OCDE]. (2018). Education at a Glance 2018: OECD Indicators. OCDE. https://www.oecd-ilibrary.org/education/education-at-a-glance-2018_eag-2018-en 
Organización de las Naciones Unidas [ONU]. (2015). Objetivos de desarrollo sostenible. Desarrollo Sostenible. https: //www.un.org/sustainabledevelopment/es/objetivos-de-desarrollo-sostenible/

Organización de las Naciones Unidas para la Educación, la Ciencia y la Cultura [UNESCO]. (2019). Descifrar el código: La educación de las niñas y las mujeres en ciencias, tecnología, ingenieria y matemáticas (STEM). UNESCO. https://unesdoc.unesco.org/ark:/48223/pf0000366649

ONU Mujeres. (2020). Efecto de la gratuidad en la incorporación de mujeres en carreras técnicas en Institutos Profesionales $y$ Centros de Formación Técnica. https://bit.ly/2UhvHuz

Petry, K. y Brackenridge, C. (2001). Women \#s Careers in Sport and Leisure Sciences/Studies in Germany: Comparison with the English situation. International Council for Health, Physical Education, Recreation, Sport and Dance, 37(3), 25-28.

Poblete, C. y Moreno, A. (2015). La mirada del género femenino en la educación física. Génesis de una historia en Chile. Géneros, 22(17), 150-166.

Porto, B. (2009). Feminización y masculinización en los estudios de maestro y educación física en Galicia. Revista de Investigación en Educación, 6(1), 50-57. https://dialnet.unirioja.es/servlet/articulo?codigo=3216105

Quintana, D. y Blazquez, N. (2017). Equidad de género en educación superior y ciencia. Agendas para América Latina y el Caribe. Universidad Nacional Autónoma de México. http://209.177.156.169/libreria_cm/archivos/pdf_1 635.pdf

Reyes-Rodríguez, L. (2007). La teoría de la acción razonada: Implicaciones para el estudio de las actitudes. Investigación Educativa Duranguense, (7), 62-72. https://dialnet.unirioja.es/servlet/articulo?codigo=2358919

Rodríguez, F., Curilem, C., Berall, F. y Almagià, A. (2017). Evaluación de la educación física escolar en Enseñanza Secundaria. Retos, 31, 76-81. https://doi.org/10.47197/retos.v0i31.49097

Ruiz-Gutiérrez, J. y Santana-Vega, L. E. (2018). Elección de carrera y género. Revista Electrónica de Investigación y Docencia (REID), (19), 9-20. https://doi.org/10.17561/reid.v0i19.3470

Sáinz, M. (2006). Aspectos psicosociales de las diferencias de género en actitudes hacia las nuevas tecnologías en adolescentes [Tesis doctoral]. Universidad de Salamanca. https://bit.ly/2TIqSdo

Scraton, S. (1995). Educación fisica de las niñas: Un enfoque feminista. Ediciones Morata.

Segobia, M. (2014). Estudiantes vespertinos: Los olvidados por la reforma educacional. El Mostrador. https://bit.ly/ $3 z \mathrm{z} 8 \mathrm{rFE}$

Serra, P. (2016). La perspectiva de género en los estudios de ciencias de la actividad física y el deporte [Tesis doctoral]. Universidad de Barcelona. https://www.tesisenred.net/handle/10803/402513

Serra, P., Soler, S., Camacho, M. J., Rey, A. y Vilanova, A. (2019a). Gendered Career Choices: Paths Toward Studying a Degree in Physical Activity and Sport Science. Frontiers in Psychology, 10. https://doi.org/10.3389/fpsyg.20 19.01986

Serra, P., Soler, S., Prat, M., Vizcarra, M.T., Garay, B. y Flintoff, A. (2016). The (in) visibility of gender knowledge in the Physical Activity and Sport Science Degree in Spain. Sport, Education and Society, 23(4). http://dx.doi.org /10.1080/13573322.2016.1199016

Serra, P., Soler, S., Vilanova, A. y Hinojosa, I. (2019b). Masculinización en estudios de las ciencias de la actividad física y el deporte. Apunts. Educación Física y Deportes, 1(135), 9-25. http://dx.doi.org/10.5672/apunts.2014-0983. es. $(2019 / 1) .135 .01$

Servicio de Información de Educación Superior [SIES]. (2010). Evolución matrícula Educación Superior de Chile. Periodo 1990-2009. Ministerio de Educación de Chile. http://bibliotecadigital.mineduc.cl//handle/20.500.12 $365 / 4609$

Simon, H. (1997). Administrative Behavior (4ta ed). Free Press.

Subsecretaría de Educación Superior de Chile. (2020). Instituciones de Educación Superior en Chile. Ministerio de Educación de Chile. https://www.mifuturo.cl/instituciones-de-educacion-superior-en-chile/ 
Carlos Matus-Castillo, et al. Masculinización de la matrícula universitaria en la carrera de Educa...

\section{INFORMACIÓN ADICIONAL}

Cómo citar: Matus-Castillo, C., Serra, P., Duclos-Bastías, D. y Castillo-Retamal, F. (2022). Masculinización de la matrícula universitaria en la carrera de Educación Física. Un análisis desde la perspectiva de género. Revista Educación, 46(1). http://doi.org/10.15517/revedu.v46i1.47576 